\title{
Ausencia de interferencias por anticuerpos heterófilos en la cuantificación de Troponina I Cardíaca, en pacientes con insuficiencia renal crónica terminal en tratamiento de hemodiálisis
}

\author{
Carmen Cecilia Almonacid $\quad{ }^{1 *}$, Jorge Cantillo Turbay $\quad{ }^{2}$, Janneth Carolina Quintana I, \\ Lorena Astrid Quiroga $\quad{ }^{1}$ y Yolima Andrea Parrado 1 \\ ${ }^{1}$ Universidad Colegio Mayor de Cundinamarca. Bogotá, Colombia. $\quad{ }^{2}$ Servicio de Terapia Renal Hospital Universitario \\ Clínica San Rafael. Bogotá, Colombia. \\ Recibido: 23-07-03; Aceptado: 05-09-03
}

\begin{abstract}
RESUMEN
El presente estudio pretende valorar el comportamiento de la adición del reactivo bloqueador de anticuerpos heterófilos (HBR) en la cuantificación de Troponina I cardíaca (TnIc) en pacientes con insuficiencia renal crónica terminal (IRCt) sometidos a tratamiento de hemodiálisis. A cada uno de los70 pacientes con IRCt en tratamiento de hemodiálisis en el Hospital Universitario Clínica San Rafael de Bogotá, se le tomó una muestra de suero y una de plasma heparinizado en la prediálisis, en las cuales se cuantificaron las concentraciones de TnIc con y sin adición de HBR, mediante el enzimoinmunoensayo Axsym. Se utilizó la prueba estadística de Wilcoxon. Los valores de TnIc en plasmas y sueros con y sin la adición de HBR no mostraron diferencias significativas (0.113 y 0.666 respectivamente), tanto en el total de los pacientes como en aquellos con enfermedad cardíaca crónica. El no haber encontrado diferencias en los valores de TnIc con y sin HBR nos lleva a pensar en la ausencia de anticuerpos heterófilos o en que el reactivo bloqueador HBR no tiene acción en esta población de pacientes.

Palabras Claves: Troponina I, cardiopatía urémica, anticuerpos heterófilos.
\end{abstract}

\section{ABSTRACT}

Absence of interferences for heterophilic antibodies in the quantification de cardiac

Troponin I, in patients with terminal chronic renal inadequacy in hemodialysis treatment.

The present study seeks to value the behavior of the addition of the blocking reagent of heterophilic antibodies (HBR), in the cardiac Troponin I quantification in patient with terminal chronicle renal inadequacy subjected to hemodialysis treatment. To each one of 70 patients with IRCt in hemodialysis treatment in the San Rafael University Hospital of Bogotá, they were taken a sample of serum and one of plasm heparanized in the pre dialysis, in which the TnIc concentrations were quantified with and without HBR addition, by means of the enzimoinmunoassay Axsym. The statistical test of Wilcoxon was used. The TnIc values in plasms and serums with and without the HBR addition didn't show significant differences ( 0.113 and 0.666 respectively), so much in the total of the patients as in those with chronic cardiac illness. Not having found differences in the TnIc values with and without HBR it takes us to think of the absence of heterophilic antibodies or in that the blocking reagent HBR doesn't have action in this population of patients.

Keywords : Troponin I, uremic cardiopatic, heterophilic antibodies. 


\section{Introducción}

Las complicaciones cardiovasculares ocupan el primer lugar como causa de muerte en la población de pacientes con daño renal crónico sometidos a tratamiento de hemodiálisis, hecho explicado por el progresivo deterioro de la unidad estructural del riñón (nefrona), que afecta directamente la función cardiaca debido al desequilibrio electrolítico y proteico y a la alteración hormonal que conlleva al desgaste mecánico del corazón (1).

El diagnóstico bioquímico de esta condición se ha complicado debido a que los marcadores cardíacos tradicionales (CK, CK-MB, LDH) e incluso algunos de la nueva generación (Troponina T y Mioglobina) son positivos en este tipo de población, independientemente de la existencia de un daño cardíaco concomitante $(2,3,4,5)$. Se crea entonces la necesidad de disponer de un marcador bioquímico altamente sensible y específico que permita la detección temprana de cardiopatías en pacientes con insuficiencia renal crónica.

En la búsqueda de este marcador, se ha comprobado que la Troponina I cardíaca cumple con muchas de las condiciones necesarias para convertirse en una herramienta diagnóstica útil en patologías miocárdicas crónicas, ya que es una proteína intracelular del miocito cardiaco liberada solo por lesión del tejido al torrente circulatorio y es codificada genéticamente por un gen específico para esta isoforma el cual no sufre reexpresión en ningún tipo de alteración músculo esquelética $(6,7,8)$.

Se ha observado que la técnica de medición presenta interferencias debido a factores inherentes a la muestra y a la misma condición fisiológica y metabólica de estos pacientes, tales como la presencia de factor reumatoideo, anticuerpos heterófilos, fibrina e ingesta de medicamentos, lo cual se ve reflejado en una baja especificidad del inmunoensayo $(9,10,11,12,13)$. En un estudio previo realizado en pacientes con IRCt hemodializados, Almonacid, et al. demostraron un
100\% de cardioespecificidad de la TnIc en el diagnóstico de lesión cardíaca en insuficiencia renal crónica y la presencia de falsos negativos, debidos probablemente a interferencias en el inmunoensayo, lo cual disminuye su sensibilidad como prueba diagnóstica (14).

Con base en lo anterior es importante adelantar estudios que neutralicen los factores de interferencia para las técnicas inmunoenzimáticas de cuantificación de Troponina I cardíaca en pacientes hemodializados, lo que facilitaría un diagnóstico más preciso y precoz de lesión miocárdica en insuficiencia renal crónica.

La presente investigación es un estudio de tipo exploratorio que tiene como objetivo establecer si el reactivo HBR bloquea o no las interferencias por anticuerpos heterófilos en la cuantificación de Troponina I cardíaca (TnIc) en pacientes hemodializados.

\section{Materiales y métodos}

Se estudiaron 70 pacientes con diagnóstico de insuficiencia renal crónica terminal (IRCt) adscritos al programa de hemodiálisis en el Hospital Universitario Clínica San Rafael de Bogotá (Colombia), con un tiempo de tratamiento no inferior a un mes y que estuvieran recibiendo 2 o 3 sesiones semanales de hemodiálisis, con valores de fosfatasa alcalina superiores a $700 \mathrm{U} / \mathrm{L}$. Se excluyeron pacientes que consumían antidepresivos tricíclicos en dosis mayores de $500 \mathrm{ng} / \mathrm{mL}$, ya que se ha comprobado que estos factores generan falsos incrementos en los valores de TnIc.

A cada paciente se le tomó una muestra de suero y una de plasma heparinizado en la pre-diálisis de la primera sesión de la semana, para un total de 140 muestras. En cada muestra recolectada se cuantificaron los niveles de TnIc con y sin la adición del reactivo HBR, mediante la tecnología Axsym de laboratorios Abbot. La presencia de patología cardíaca se comprobó mediante ecocardiograma.

En la descripción se utilizaron valores de agrupación y dispersión para las variables numéricas y pro- 
porciones para las variables cualitativas. Se emplearon las correlaciones de Pearson o Spearman según variables numéricas u ordinales, con un valor de significancia de $\mathrm{p}=0.05$. Para el análisis de prueba diagnóstica se realizaron tablas de 2x2 y se utilizó el programa Epidat 2.1 1997. En el análisis de Troponina I cardíaca (con y sin reactivo HBR) se aplicó el test de Wilcoxon. El programa estadístico fue SPSS versión 9 del 2000.

\section{Resultados}

Descripción del grupo de pacientes:

En los 70 pacientes analizados la mediana de edad fue de 50 años (13 -78 años) y de ellos, 38 pacientes (54.3\%) fueron de sexo masculino.

El tiempo promedio de hemodiálisis fue de 16 meses (1-20 meses) (Tabla 1). Del total de la población analizada, 23 pacientes (32.9\%), estuvieron vinculados previamente al programa de diálisis peritoneal ambulatoria continua (CAPD).

Se observó que $32(45.7 \%)$ de los pacientes con insuficiencia renal crónica terminal tenían como etiología una nefropatía.

$39(55.7 \%)$ de los pacientes tenían enfermedad cardíaca crónica comprobada por ecocardiograma.

Durante el estudio fallecieron 2 pacientes (Tabla 1).

\section{Comportamiento de la Troponina I cardíaca}

El valor promedio para TnIc en las 140 muestras de suero procesadas sin la adición de reactivo HBR fue de $0.139 \mathrm{ng} / \mathrm{mL}$ con una DS de $0.448 \mathrm{ng} / \mathrm{mL}$ y una mediana de $0 \mathrm{ng} / \mathrm{mL}(0-2.6 \mathrm{ng} / \mathrm{mL})$; mientras que al procesarlas con el reactivo HBR se obtuvo una media de $0.087 \mathrm{ng} / \mathrm{mL}$ con una DS de $0.258 \mathrm{ng} / \mathrm{mL}$ y una mediana de $0 \mathrm{ng} / \mathrm{mL}(0-1.3 \mathrm{ng} / \mathrm{mL})$ (Figuras 1 y 2 Tabla 2).

El valor promedio para TnIc encontrado en las 140 muestras de plasma analizadas sin HBR fue de 0.087 $\mathrm{ng} / \mathrm{mL}$ con una DS de $0.203 \mathrm{ng} / \mathrm{mL}$ y una mediana de $0.0 \mathrm{ng} / \mathrm{mL}(0-1.1 \mathrm{ng} / \mathrm{mL})$; con la adición de HBR, el

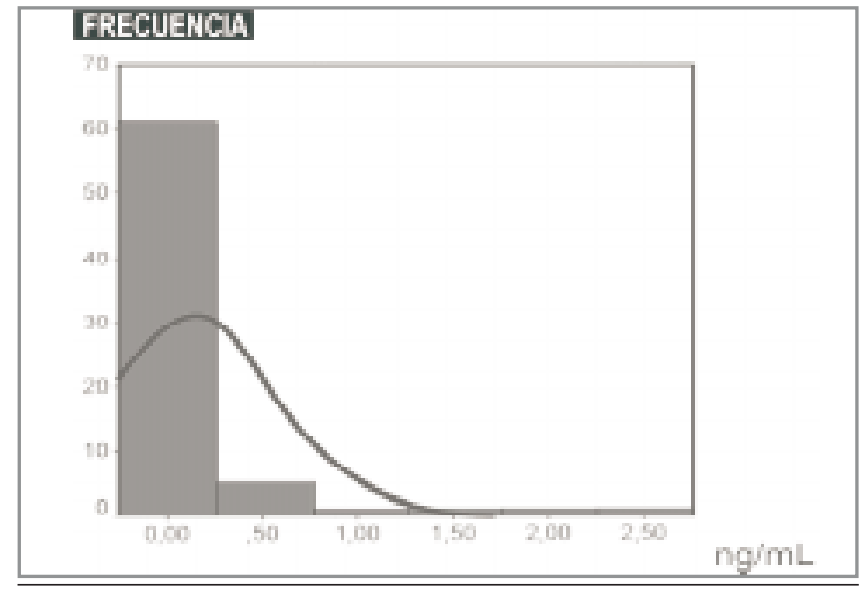

Figura 1: Distribución de TnIc en suero sin adición de HBR.

\section{FRECUENCIA}

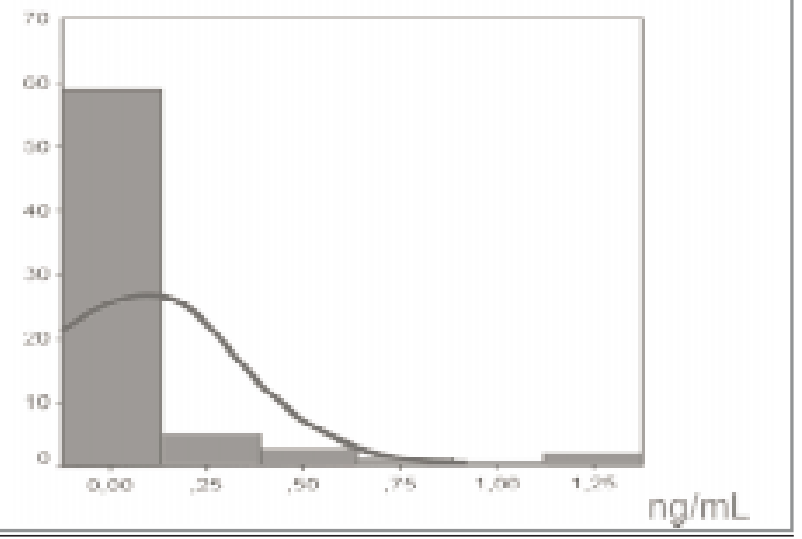

Figura 2: Distribución de TnIc en suero con adición de HBR.

valor medio obtenido fue de $0.110 \mathrm{ng} / \mathrm{mL}$ con una $\mathrm{DS}$ de $0.240 \mathrm{ng} / \mathrm{mL}$ y una mediana de $0.0 \mathrm{ng} / \mathrm{mL}$ (0-1.2ng/ $\mathrm{mL}$ ). La distribución de TnIc en plasmas con y sin adición de HBR fue igual que en suero.

Según la prueba de Wilcoxon no hubo diferencia estadísticamente significativa entre los valores de TnIc en los sueros y plasmas procesados con y sin adición de HBR ( $p=0.666$ y $p=0.113$ respectivamente) (Tabla 2).

En los 26 pacientes con enfermedad crónica (20 hipertrofia ventricular izquierda, 5 con cardiopatía dilatada), los valores en suero y plasma de TnIc con $y$ sin aditivo no mostraron una diferencia estadísticamente significativa $(p=0.666$ y $p=0.113$ respectivamente) (Tabla 2). 
TIEMPO EN DIÁLISIS FRECUENCIA PORCENTAJE Menos de 1 año

1 a 3 años

23

32.9

3.1 a 5 años

41.4

5.1 a 8 años

Más de 8 años

No conocido

TOTAL

29

12.9

7.1

4.3

1.4

100

ETIOLOGÍA

Nefropatía hipertensiva

25.7

Nefropatía diabética

14.3

Nefropatía Lúpica

5.7

Riñón poliquístico

Desconocida

2.8

28.6

Glomerulonefritis

Urotapía obstructiva

Otras

TOTAL

DATOS

ECOCARDIOGRÁFICOS

Cardiopatía hipertensiva

Cariopatía dilatada

Hipertrofia ventricular izquierda

Insuficiencia cardíaca congestiva

Enfermedad coronaria

No presentó alteración

Sin ecocardiograma

FALLECIMIENTO

Nó

13

5

20

1

3

2

26

2

68

2.9

97.1
Tabla 1: Descripción del grupo de pacientes.

\section{Discusión}

La presente investigación no mostró diferencias en los valores de TnIc en los sueros y plasmas procesados con y sin adición del reactivo HBR. Esto nos lleva a concluir que los falsos negativos encontrados en la valoración de TnIc en este tipo específico de población, no son producto de un alto título de anticuerpos heterófilos, sino de otro tipo de interferencias, o son debidas a que el reactivo bloqueador HBR no actúa en este tipo de patología. Diversos estudios han demostrado que los anticuerpos heterófilos presentes en el plasma pueden unirse a los anticuerpos de captura utilizados en varios de los inmunoensayos para la cuantificación de Troponina I cardíaca, generando interferencias que disminuyen la sensibilidad de las técnicas. El pre-tratamiento de las muestras con el reactivo bloqueador de anticuerpos heterófilos (HBR) ha sido ensayado con éxito como un método para minimizar el efecto de estos anticuerpos en poblaciones diferentes a pacientes con insuficiencia renal (13).

La manifestación clínica más frecuente de daño cardíaco en pacientes sometidos a hemodiálisis es la falla cardíaca congestiva asociada con falla sistólica, hipertrofia ventricular izquierda y enfermedad isquémica cardíaca $(15,16)$. Diversos estudios demuestran que los extremos amino y carboxilo terminal de la proteina de TnIc son susceptibles de sufrir proteólisis y que esta alteración está relacionada con la degradación isquémica del tejido (12). Se han reportado 11 productos modificados de TnIc entre los que se encuentran los complejos con Troponina C (IC), troponina T (ITC), las formas oxidadas, reducidas y fosforiladas y la formación de bisulfuros entre cistenas $(12,17)$. La presencia de esta variedad de formas moleculares dificulta la cuantificación ya que los anticuerpos utilizados en los inmunoensayos deben reconocer preferiblemente los epitopes que están localizados en la parte estable de la molécula y no los susceptibles de modificación (18). En nuestro estudio, pacientes con alteraciones cardíacas comprobadas mediante ecocardiograma no mostraron modificación en los niveles de TnIc. Esto puede ser debido a un incremento en la proteólisis de la molécula de TnIc en este tipo específico de población por la degradación continua y profunda del miocardio. Esta variedad de formas circulantes no pueden ser captadas por los anticuerpos de captura utilizados en la técnica de cuantificación, lo que genera resultados falsos negativos.

Los estudios reportados sobre el comportamiento de la TnIc en la pre y postdiálisis son pocos $(9,14)$. Un estudio previo realizado en pacientes con IRCt sugirió que la cuantificación de los niveles de TnIc en 


\begin{tabular}{|c|c|c|c|c|c|c|}
\hline & $\pi$ & Motla & 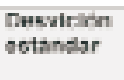 & Minims & mixims & $\begin{array}{l}\text { Pruptas de } \\
\text { wilesung }\end{array}$ \\
\hline $\begin{array}{l}\text { Sump sin } \\
\text { adioion } \\
\text { HER }\end{array}$ & 70 & 0.139 & $0 \leq 45$ & 0 & 26 & $z=0.43 z$ \\
\hline $\begin{array}{c}\text { Euero } \\
\text { cuil } \\
\text { adieion } \\
\text { HMR }\end{array}$ & 70 & 0057 & 0.258 & 0 & 13 & $p-0.66$ \\
\hline $\begin{array}{l}\text { Plasith } \\
\text { Ein } \\
\text { dulibiul } \\
\text { HBR }\end{array}$ & $\pi$ & dogr & 0203 & d & 1.1 & $\begin{array}{l}z=-1.54 \\
\Gamma=0.113\end{array}$ \\
\hline $\begin{array}{l}\text { Pasms } \\
\text { con } \\
\text { stlolon } \\
\text { HBR }\end{array}$ & 70 & 0.110 & 0240 & 0 & 12 & \\
\hline
\end{tabular}

Tabla 2: Valores de Tnlc en suero y plasma con y sin HBR.

la postdiálisis tiene mayor valor diagnóstico que los de la prediálisis (14). Nuestros datos coinciden con este informe ya que la no elevación de las concentraciones de TnIc prediálisis en pacientes con cardiopatía urémica, demuestran menor especificidad de las valoraciones de TnIc prediálisis para la detección de daño miocárdico en pacientes hemodializados. Esta situación puede deberse a las altas concentraciones de metabolitos presentes en la sangre de los pacientes insuficientes renales crónicos que son removidos a través de la diálisis y que podrían estar interfiriendo con el inmunoensayo. Por lo tanto recomendamos adelantar un nuevo proyecto en el que se tengan en cuenta como variables las concentraciones de estos metabolitos en sangre durante la pre y la postdiálisis.

\section{Referencias}

1. Parfrey P, et al. The clinical epidemiology of cardiac disease in chronic renal failure. Journal of the American Society of Nephrology 1999;10:1606-15.

2. Mac Laurin M, et al. Cardiac troponin I, cardiac troponin T, and creatine kinase MB in dialys patients without ischemic heart disease: Evidence of cardiac Troponin T expression in skeletal muscle. Clin Chem 1997;43(6):976-82.
3. Montgomery J, et al. Comparison of serum and EDTA plasma for CK-MB, myoglobin and cardiac Troponin I (cTnI) using the Beckman Access immunoassay Analyzer. Clin Chem 1998;44(6):A141.

4. Datta P. Bayer Corporation, Diagnostic Division. ACS 180 cardiac Troponin I (cTnI) and myoglobin assays are free from rheumatoid factor interference. Clin Chem 1999;45(6):A136.

5. Wayand D, et al. Cardiac Troponin T and I in EndStage Renal Failure. Clin Chem 2000;46:1345-50.

6. Granier C, et al. Human cardiac Troponin I: Precise identification of antigenic epitopes and prediction of secondary structure. Clin Chem 1998;44(3):487-93.

7. Coudry L, et al. The Troponins. Arch Intern Med 1998;158:1173-80.

8. Panteghini M. Acute Coronary Syndrome. Chest 2002;122(4):1428-35.

9. Willging S, et al. Specificity of cardiac Troponins I and $\mathrm{T}$ in Renal Disease. Clin Chem Lab Med 1998;36(2):87-92.

10. Hohnadel D, et al. Alternative samples for Troponin I testing. Clin Chem 1998;44(6):A120.

11. Fitzmaurice T, et al. False increase for cardiac Troponin I with heterophilic antibodies. Clin Chem 1998;44(10):22124.

12. Qinwei S, et al. Degradation of cardia Troponin I in serum complicates comparisons of cardiac troponin I assays. Clin Chem 1999;45(7):1018-25.

13. Gerhardt W, et al. Troponin T and I assays show decreased concentrations in heparin plasma compared with serum: lower recoveries in early than in late phases of myocardial injury. Clin Chem 2000;46:817-21.

14. Almonacid C, et al. Comportamiento y valor pronóstico de la Troponina I cardiaca en Insuficiencia Renal Crónica: un estudio en 69 pacientes de hemodiálisis. Acta Médica Colombiana 2002;27(5):306.

15. Hartnert J, et al. Congestive hart failure in dialysis patients: prevalence, incidence, prognosis and risk factors. Kidney International 1995;47:884-90.

16. Stoffel M, et al. Troponin I is a better prognostic parameter of cardiovascular events in asymtomatic patients on haemodialysis than troponin T. Nephrol Dial Transplant 2000;15:1259-60.

17. Labugger R, et al. Extensive Troponin I and T modification detected in serum from patients with acute myocardial infarction. Circulation 2000;102:1221-6.

18. Katrukha A, et al. Degradation of cardiac troponin I: implication for reliable immunodetection. Clin Chem 1998;44(12):2433- 40. 